\title{
The role of posttraumatic stress symptoms on chronic pain outcomes in chronic pain patients referred to rehabilitation
}

This article was published in the following Dove Press journal: Journal of Pain Research

\author{
Sophie Lykkegaard Ravn ${ }^{1,2}$ \\ Henrik Bjarke Vaegter ${ }^{3,4}$ \\ Thomas Cardel' \\ Tonny Elmose Andersen' \\ 'Department of Psychology, \\ University of Southern Denmark, \\ Odense, Denmark; ${ }^{2}$ The Specialized \\ Hospital for Polio and Accident \\ Victims, Roedovre, Denmark; ${ }^{3}$ Pain \\ Research Group, Pain Center South, \\ Department of Anesthesiology and \\ Intensive Care Medicine, Odense \\ University Hospital, Odense, \\ Denmark; ${ }^{4}$ Department of Clinical \\ Research, University of Southern \\ Denmark, Odense, Denmark
}

Objectives: Posttraumatic stress symptoms (PTSS) are highly prevalent in chronic pain patients and may affect pain symptomatology negatively, but there is still a great need to explore exactly how this occurs. Therefore, this study investigated differences in pain intensity, pain-related disability, and psychological distress between chronic pain patients not exposed to a trauma, patients exposed to a trauma with no PTSS, and patients exposed to a trauma with PTSS. Moreover, the moderating effects of PTSS on the associations between pain intensity and pain-related disability and psychological distress were investigated.

Methods: In this cross-sectional cohort study, data were consecutively collected over the course of a year in patients with chronic non-malignant pain referred for multidisciplinary pain rehabilitation at a Danish university hospital pain center using questionnaires assessing pain, pain-related disability, PTSS, anxiety, and depression.

Results: The final sample consisted of 682 chronic pain patients, who were divided into three subgroups (no trauma, 40.6\%; trauma/no PTSS, 40.5\%; trauma/PTSS, 18.9\%). Chronic pain patients with PTSS reported significantly higher levels of pain intensity, pain-related disability, depression, and anxiety compared to chronic pain patients without a trauma and chronic pain patients without PTSS. Moreover, PTSS significantly moderated the associations between pain intensity and pain-related psychosocial disability, depression, and anxiety.

Conclusion: These results highlight the importance of assessing PTSS in chronic pain patients and suggest that PTSS have a specific influence on the association between pain intensity and more psychosocial aspects of the pain condition.

Keywords: chronic pain, posttraumatic stress symptoms, PTSS, PTSD, distress, pain-related disability

\section{Introduction}

Posttraumatic stress disorder (PTSD) or symptoms hereof are common in chronic pain patients with approximately $25 \%$ of patients admitted to pain clinics in Scandinavia fulfilling the DSM-IV diagnostic criteria for possible PTSD. ${ }^{1,2}$ Posttraumatic stress symptoms (PTSS) represent symptoms of PTSD that are not necessarily compliant with a full diagnosis. According to DSM-IV, PTSD is a psychological reaction to a traumatic event with symptoms of intrusion, avoidance, and hyperarousal. ${ }^{3}$ Mutual maintenance between pain and PTSD has been proposed with the conditions influencing and exacerbating each other through an array of physiological, cognitive, behavioral, and affective mechanisms. ${ }^{4}$ Recent reviews supported this idea by demonstrating evidence of mutual influence ${ }^{5}$ and reciprocal interdependence ${ }^{6}$ between pain and PTSD, hence
Correspondence: Sophie Lykkegaard Ravn

Department of Psychology, University of Southern Denmark, Campusvej 55, DK-5230 Odense M, Denmark

Tel +45268 I 9022

Email slravn@health.sdu.dk 
highlighting the hypothesis that PTSS can have a negative effect on pain outcomes as well as vice versa.

Across chronic pain conditions, comorbid PTSS among chronic pain patients has consistently been found to be associated with increased pain and psychological distress. ${ }^{7-9}$ Increased levels of pain-related disability have also been associated with comorbid PTSS, ${ }^{8,9}$ although some studies only reported increased psychosocial disability and not physical disability. ${ }^{1,10}$ However, it remains unclear whether the more severe symptomatology among chronic pain patients with comorbid PTSS is related to the trauma exposure per se or the prolonged reaction to it (i.e., PTSS/PTSD). To our knowledge, only two studies have previously investigated this, demonstrating that chronic pain patients with comorbid PTSS reported higher levels of pain intensity, pain-related disability, and psychological distress ${ }^{11}$ and higher levels of pain interference, kinesiophobia, and psychological distress ${ }^{12}$ compared with chronic pain patients not exposed to a trauma and patients exposed to trauma, but with no PTSS among chronic back pain patients and a mixed chronic pain sample, respectively. This indicates that PTSS, and not the traumatic exposure in itself, is related to a more severe pain profile. However, studies ought to replicate these findings and build upon this by investigating the specific influence of PTSS on pain-related outcomes and their interrelations among chronic pain patients referred to treatment.

Looking more into the specific effects of PTSS, RuizPárraga and López-Martínez ${ }^{11}$ found that PTSS moderated the associations between, among others, fear-avoidancevariables and pain intensity. However, the moderating effect of PTSS on the clinically important associations between pain intensity and disability and distress was not tested. Indeed, Roth et al. ${ }^{13}$ found that PTSS contributed significantly to depressive symptoms, pain intensity, and pain-related disability after controlling for anxiety among chronic pain patients and highlighted the need to address unresolved PTSS in chronic pain rehabilitation. Likewise, several other studies have reported on PTSS in the interplay between pain, disability, depression, and anxiety. ${ }^{14-16}$ Despite increasing research in the field, there is still a great need to explore exactly how comorbid PTSS is associated with pain-related outcomes among chronic pain patients seeking rehabilitation for their pain. As the previously mentioned studies found that chronic pain patients with comorbid PTSS have more severe symptomatologies, it is highly relevant to go a step further and investigate the potential moderating effects of PTSS on some of the important clinical outcomes (eg, pain, related disability, and distress) and their interrelations presented in the clinic by the patients. If comorbid PTSS does indeed moderate the relationships of these outcomes, this can be of great clinical importance. Therefore, further examinations of the potential moderating effects of PTSS on associations between pain intensity and related outcomes such as disability and distress among large cohorts of chronic pain patients referred for multidisciplinary treatment are needed.

Thus, the overall aim of this study was to investigate how comorbid PTSS influenced pain-related outcomes and their interrelations. This was done through two objectives. The first objective was to investigate whether levels of pain intensity, pain-related disability, and psychological distress differed between chronic pain patients not exposed to a trauma, patients exposed to trauma with no PTSS, and patients exposed to trauma with comorbid PTSS in order to replicate two earlier studies. The second objective was to investigate whether PTSS moderated the effects of pain intensity on pain-related disability and psychological distress. It was hypothesized that chronic pain patients with comorbid PTSS would present with higher levels of pain, pain-related disability, and psychological distress compared with the other two groups, and that PTSS would moderate all associations between pain intensity, disability, and psychological distress.

\section{Materials and methods Participants and procedure}

The context for this cross-sectional study was a secondary setting, multidisciplinary pain center at a large University Hospital in Denmark that assesses and treats patients with chronic non-malignant pain. After referral to this multidisciplinary pain clinic, but prior to their initial consultation, all patients were invited to answer a set of questions about their clinical characteristics and adaptations to pain via several questionnaires. The questionnaires were completed at home prior to the first consultation. Data were consecutively collected from 760 chronic pain patients in the period from February 2014 to February 2015. The Danish Data Protection Agency approved the data collection, and the conduct of this study complied with the Declaration of Helsinki. As treatment was not affected by participation in the study, under Danish law and according to the guidelines from the National Committee on Health Research Ethics, this study did not need ethics approval and written informed consent for participation was not required.

\section{Outcome measures}

Initially, all participants reported on descriptive characteristics, which included age, gender, relationship status, highest 
level of education, basis of support, number of pain locations, and pain duration.

\section{Pain intensity}

Pain intensity was measured with a numeric rating scale that is designed to measure pain intensity. ${ }^{17,18}$ The scale is a numeric 11-point scale that ranges from 0 (no pain) to 10 (worst imaginable pain). In this project, participants were asked to rate "average pain during the last 24 hours" as used in the Brief Pain Inventory, a tool that has been found to be both reliable and valid. ${ }^{19}$

\section{Pain-related disability}

Pain-related disability was measured with Pain Disability Questionnaire. ${ }^{20}$ The scale is designed to measure pain-related dysfunction among people with chronic musculoskeletal conditions. The scale has 15 items and each item is rated from 0 to 10 on a numeric scale where 0 is no disability and 10 is worst imaginable disability. The scale consists of two subscales measuring physical (nine items) and psychosocial (six items) pain-related disabilities. The scale has good psychometric properties and has been validated among several pain populations. Among chronic musculoskeletal pain patients, the scale has great internal reliability $(\alpha=0.96)$ and consistency over time $(r=0.94, p<0.01) .{ }^{20}$ For the purpose of this study, the subscales were used as outcomes. The physical component was found to have good internal reliability $(\alpha=0.86)$, while the psychosocial component had acceptable internal reliability $(\alpha=0.71)$.

\section{Psychological distress}

The Hospital Anxiety and Depression Scale ${ }^{21}$ was used to assess levels of depression and anxiety. The scale has been validated in psychiatric assessment settings with high, positive correlations for both depression $(r=0.70, p<0.001)$ and anxiety $(r=0.74, p<0.001) .{ }^{21}$ The scale consists of 14 items that measure the subdomains of depression (seven items) and anxiety (seven items). The items are answered on a 4-point Likert-scale (0-3). Hence, each subscale has a scoring span from 0 to 21 points. A score above 11 indicates the presence of anxiety/depression. ${ }^{21}$ Test-retest reliability was good for both the anxiety subscale $(r=0.89, p<0.05)$ and the depression subscale $(r=0.92, p<0.05),{ }^{21}$ and likewise, Crawford et al. ${ }^{22}$ found good internal consistency on both scales $(\alpha=0.82 ; \alpha=0.77)$. Good internal reliability was also found in the current study for both anxiety $(\alpha=0.84)$ and depression $(\alpha=0.82)$.

\section{PTSS}

Initially, patients were asked to answer whether or not they had been exposed to a traumatic event. As a part of this, different examples of traumas (eg, accidents, physical or mental violence, disasters, serious illness, death of a near relative) were provided. If yes, PTSS were assessed using the PTSD- $8 .{ }^{23}$ The scale consists of eight items assessing the core clusters of PTSS as outlined by DSM-IV-TR ${ }^{3}$ and the proposed ICD-11-model; ${ }^{24}$ re-experiencing (four items; $\alpha=0.88$ ), avoidance (two items; $\alpha=0.92$ ), and hyperarousal (two items; $\alpha=0.92$ ). ${ }^{23}$ Items are answered on a 4-point Likert scale (1="not at all" to $4=$ "for the most part") with a higher score indicating higher levels of posttraumatic symptoms. To fulfill the criteria for possible PTSD (or as labeled in the present study PTSS), at least one item on each cluster has to be $\geq 3$. The scale has good psychometric properties and has been, among others, validated in a sample of whiplashpatients with acceptable internal reliability $(\alpha=0.74)$ and good consistency over time $(r=0.82, p<0.001) .{ }^{23}$ Further, a recent study established a good overall accuracy of the PTSD- 8 against a diagnostic interview among chronic pain patients. ${ }^{25}$ In the current study, there was good internal reliability $(\alpha=0.88)$.

\section{Subdivision of groups}

For the purpose of this study, three subgroups were identified based on the ratings of the PTSD-8. Patients with no trauma were the ones answering "no" to the initial trauma question (group 1). These patients did not fill out the PTSD-8 questionnaire, as the index trauma is a fundamental criterion to be able to speak of PTSS. Patients with a trauma, but no PTSS were the ones answering, "yes" to the initial trauma question, but not reporting clinically relevant levels of PTSS based on the scoring key (less than one item $\geq 3$ on each cluster; group 2). Finally, patients with a trauma and PTSS were the ones answering "yes" to the initial trauma question and reporting clinical relevant levels of PTSS based on the scoring key (at least one item $\geq 3$ on each cluster; group 3 ).

\section{Statistics}

To begin with, data were screened for missing values. Cases that had above $20 \%$ missing items or were missing a complete scale were excluded (Figure 1). To decide whether data had missing values in a random pattern, analyses of differences between these groups in all of the variables were done using Little's Missing Completely at Random test. ${ }^{26}$ Missing values were then imputed using the Expectation-Maximization method. ${ }^{27}$ 


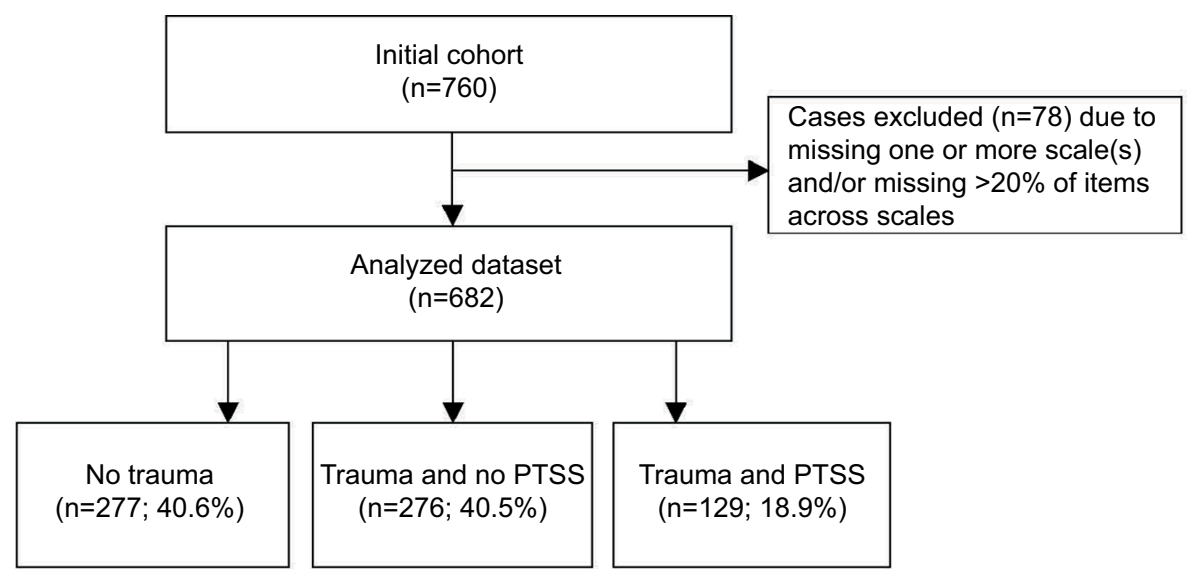

Figure I Study flow chart.

Abbreviation: PTSS, posttraumatic stress symptoms.

Before testing the hypothesis, the sample was split into the subgroups and was described and compared in terms of demographic variables using percentages and Chi-square tests for categorical variables and means, standard deviations, and one-way analysis of variance for continuous variables. To test the first hypothesis, between-group differences in pain intensity, pain-related disability, and psychological distress were tested using ANOVAs. In total, 31 comparisons were made using these tests for these two purposes. Therefore, the $p$-value was adjusted accordingly $(p \leq 0.05 / 31)$ in order to address the risk of Type 1 errors. Hence, $p$-values $\leq 0.002$ were considered significant. Post hoc analyses were conducted to reveal the nature of significant effects. For this purpose, as group sizes were not equal, the post hoc test of Gabriel was used. ${ }^{28}$ Further, effect sizes of group differences on continuous variables were calculated manually post hoc and were calculated by taking the square root of eta squared.$^{28}$ Effect sizes were classified as small $(r=0.10)$, medium $(r=0.30)$, and large $(\mathrm{r}=0.50) .^{28}$

In order to test the second hypothesis, four moderation tests were conducted to test PTSS as a moderator of the effect of pain intensity on pain-related disability (psychosocial and physical, respectively) and psychological distress (depression and anxiety, respectively). For this purpose, PTSS was used as a binary variable $(0=$ no PTSS, $1=$ PTSS $)$. The analyses were done using the SPSS macro PROCESS, which is a bootstrapped regression-based method using mean-centered multiple hierarchical regression analyses. ${ }^{28,29}$ Moderation is the effect of one variable on the relationship of two others, ${ }^{28}$ and hence, moderation is present when the nature of a relationship between the independent and the dependent variables changes as a function of the moderator.

\section{Results}

\section{Sample characteristics and correlations of variables}

Six hundred and eighty-two patients were included in the analyses and were split into the three subgroups: no trauma (group 1; 40.6\%), trauma and no PTSS (group 2; 40.5\%), and trauma and PTSS (group 3; 18.9\%). Demographic details for the groups were reported (Table 1), and all outcomerelated variables were significantly and positively correlated ( $p<0.001$; Table 2$)$. When comparing the subgroups in terms of demographic details, there were no significant group differences in gender, age, highest level of education, basis of support, relationship status, pain medication, other medication, types of pain, nor pain duration (Table 1). However, a significant difference was found for number of pain locations and shoulder pain with post hoc analyses revealing that patients with no trauma had significantly fewer pain locations and a lower percentage reported shoulder pain compared with the two other groups $(p \leq 0.001)$.

\section{Group differences in pain intensity, pain-related disability, and psychological distress}

Significant group differences were found in the ANOVAs of pain intensity $(F(2,679)=9.019, p<0.001)$, psychosocial pain-related disability $(F(2,679)=33.203, p<0.001)$, physical pain-related disability $(F(2,679)=7.023, p=0.001)$, depression $(F(2,679)=38.237, p<0.001)$, and anxiety $(F(2,679)=58.881$, $p<0.001$ ) (Table 1). Post hoc tests showed significantly higher levels of pain intensity, psychosocial pain-related disability, physical pain-related disability, depression, and anxiety in 


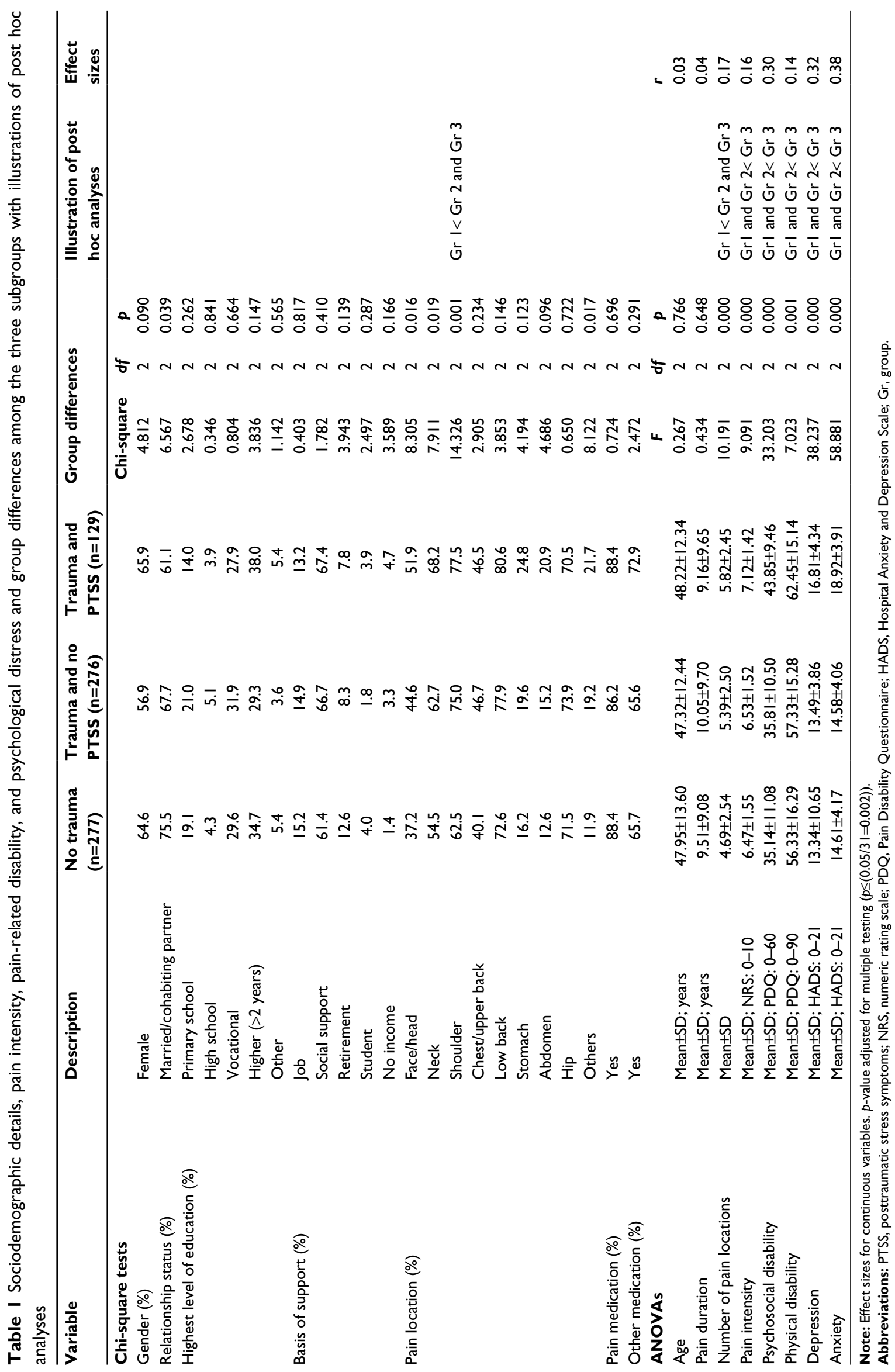


patients with PTSS (group 3) compared to the other two groups $(p<0.001)$.

\section{PTSS as a moderator of the associations between pain intensity and outcomes}

The association between pain intensity and psychosocial disability was significantly moderated by PTSS (Table 3 and Figure 2; $b=2.45,95 \%$ CI $[0.98,3.91], t=3.29, p=0.011$ ). Higher pain intensity was associated with higher levels of psychosocial disability both when PTSS was and was not present, but pain intensity in relation to clinical levels of PTSS was associated with higher levels of psychosocial disability compared to when PTSS was not present.

The association between pain intensity and physical disability was not significantly moderated by PTSS (Table 3 ; $b=1.86,95 \%$ CI $[-0.55,4.26], t=1.52, p=0.129)$. Higher

Table 2 Correlations for all outcome variables $(n=682)$

\begin{tabular}{llllll}
\hline Variables & I & $\mathbf{2}$ & $\mathbf{3}$ & $\mathbf{4}$ & $\mathbf{5}$ \\
\hline $\begin{array}{l}\text { I. Pain intensity } \\
\text { 2. Psychosocial pain-related }\end{array}$ & 0.342 & & & & \\
$\quad$ disability & & & & & \\
$\begin{array}{l}\text { 3. Physical pain-related } \\
\quad \text { disability }\end{array}$ & 0.448 & 0.552 & & & \\
4. Depression & & & & & \\
5. Anxiety & 0.155 & 0.566 & 0.308 & & \\
$\begin{array}{l}\text { 6. Posttraumatic stress } \\
\text { symptoms }\end{array}$ & 0.167 & 0.520 & 0.254 & 0.680 & \\
\hline
\end{tabular}

Note: All correlations are significant $(p<0.001)$. pain intensity was associated with higher levels of physical disability, but the nature of this relationship did not change as a function of PTSS.

The association between pain intensity and depression was significantly moderated by PTSS (Table 3 and Figure 3; $b=0.855,95 \% \mathrm{CI}[0.278,1.43], t=2.91, p=0.004)$. The analyses revealed that higher pain intensity was associated with higher levels of depression both when PTSS was and was not present, but that pain intensity in relation to clinical levels of PTSS was associated with higher levels of depression compared to when PTSS was not present.

The association between pain intensity and anxiety was significantly moderated by PTSS (Table 3 and Figure 4; $b=0.771,95 \%$ CI $[0.173,1.37], t=2.53, p=0.012$ ), suggesting that the presence of PTSS did change the nature of the relationship between pain intensity and anxiety.

\section{Discussion}

\section{Summary of findings}

The aim of the current study was to investigate the role of PTSS in pain-related outcomes and their interrelations in a large cohort of chronic pain patients referred to a multidisciplinary pain clinic. Chronic pain patients with PTSS reported significantly higher levels of pain intensity, pain-related disability (psychosocial and physical), depression, and anxiety compared with chronic pain patients without a trauma and chronic pain patients with a trauma, but without clinical levels

Table 3 PTSS as a moderator of the associations between pain intensity and outcomes

\begin{tabular}{|c|c|c|c|c|c|}
\hline Dependent variable & Independent variables & $b[95 \% \mathrm{Cl}]$ & SE $b$ & $t$ & $p$ \\
\hline \multicolumn{6}{|l|}{ Psychosocial disability } \\
\hline & Constant & 38.06 [37.08-39.03] & 0.495 & 76.90 & $p<0.001$ \\
\hline & PTSS & $6.26[4.30-8.21]$ & 0.996 & 6.28 & $p<0.001$ \\
\hline & Pain intensity & $2.12[1.35-2.89]$ & 0.393 & 5.41 & $p<0.001$ \\
\hline & PTSS $\times$ pain intensity & $2.45[0.98-3.91]$ & 0.745 & 3.29 & $p=0.011$ \\
\hline \multicolumn{6}{|l|}{ Physical disability } \\
\hline & Constant & $58.72[57.31-60.13]$ & 0.717 & 81.86 & $p<0.001$ \\
\hline & PTSS & $2.13[-1.05-5.30]$ & 1.615 & 1.32 & $p=0.188$ \\
\hline & Pain intensity & $4.38[3.23-5.52]$ & 0.582 & 7.53 & $p<0.001$ \\
\hline & PTSS $\times$ pain intensity & $1.86[-0.55-4.26]$ & 1.222 & 1.52 & $p=0.129$ \\
\hline \multicolumn{6}{|l|}{ Depression } \\
\hline & Constant & $14.43[14.04-14.82]$ & 0.198 & 73.02 & $p<0.001$ \\
\hline & PTSS & $2.86[2.03-3.72]$ & 0.429 & 6.71 & $p<0.001$ \\
\hline & Pain intensity & $0.442[0.178-0.707]$ & 0.135 & 3.29 & $p=0.001$ \\
\hline & PTSS $\times$ pain intensity & $0.855[0.278-1.43]$ & 0.294 & 2.91 & $p=0.004$ \\
\hline \multicolumn{6}{|l|}{ Anxiety } \\
\hline & Constant & $15.86[15.48-16.26]$ & 0.198 & 80.35 & $p<0.001$ \\
\hline & PTSS & $4.01[3.21-4.81]$ & 0.401 & 9.84 & $p<0.001$ \\
\hline & Pain intensity & $0.288[0.004-0.572]$ & 0.145 & 1.99 & $p=0.047$ \\
\hline & PTSS $\times$ pain intensity & $0.77 \mid[0.173-1.37]$ & 0.305 & 2.53 & $p=0.012$ \\
\hline
\end{tabular}

Note: PTSS is a binary variable (yes/no).

Abbreviation: PTSS, posttraumatic stress symptoms. 


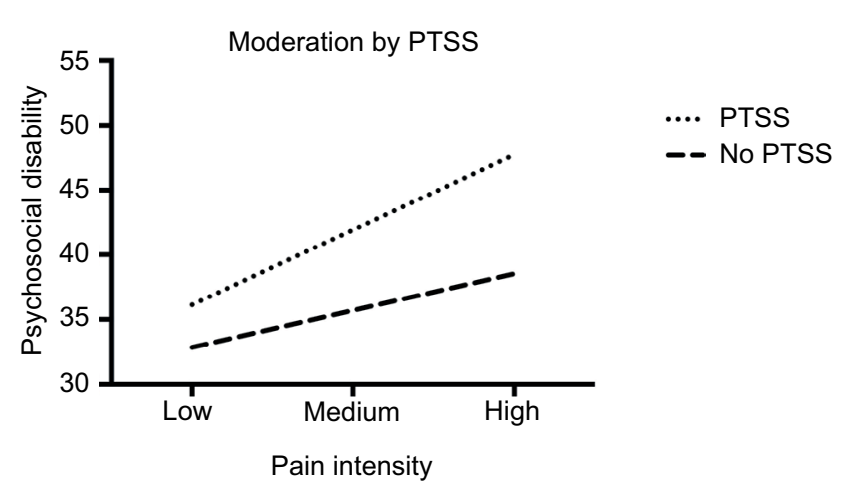

Figure $\mathbf{2}$ The association between pain intensity and psychosocial disability moderated by PTSS.

Abbreviation: PTSS, posttraumatic stress symptoms.

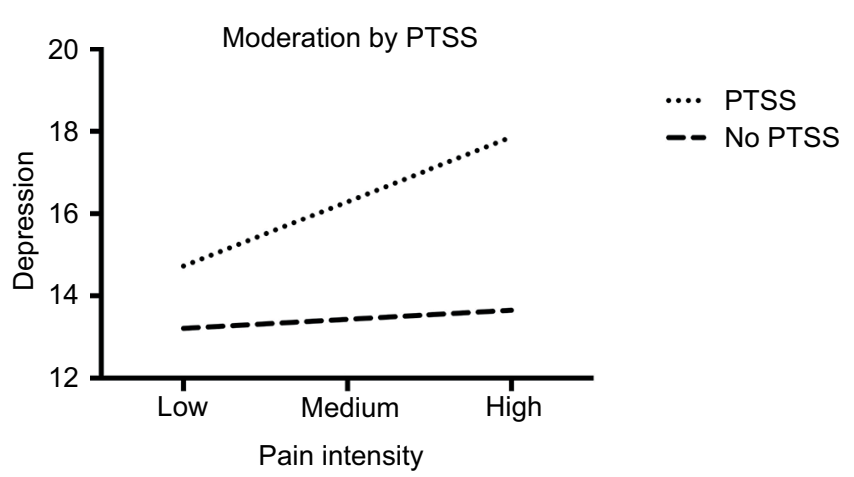

Figure 3 The association between pain intensity and depression moderated by PTSS.

Abbreviation: PTSS, posttraumatic stress symptoms.

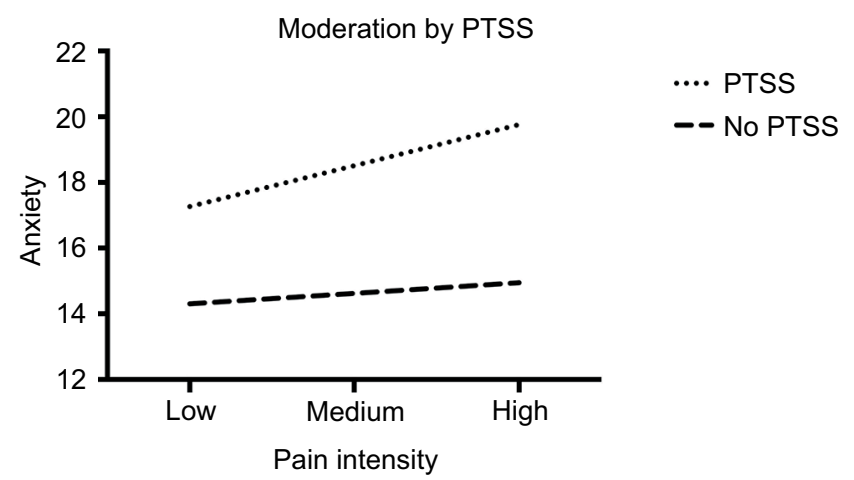

Figure 4 The association between pain intensity and anxiety moderated by PTSS. Abbreviation: PTSS, posttraumatic stress symptoms.

of PTSS, suggesting that PTSS and not exposure to trauma per se is related to a more severe clinical pain profile. Moreover, PTSS significantly moderated the associations between pain intensity and pain-related psychosocial disability, depression, and anxiety, but not pain-related physical disability. These findings indicate that whenever PTSS are present, the relationship between pain and psychological distress will be stronger compared to cases where PTSS are not present, while this is not the case for physical pain-related disability.

\section{Group differences in terms of pain, disability, and psychological distress}

Pain intensity, pain-related disability, and psychological distress were significantly increased in patients with PTSS compared to patients without a trauma and patients without relevant levels of PTSS. This is in agreement with the findings of Ruiz-Párraga and López-Martínez ${ }^{11}$ who, in a sample of 346 back pain patients, found that patients with PTSS reported significantly higher levels of pain, pain-related disability, and psychological distress. Similarly, Åkerblom et al. ${ }^{12}$ found enhanced levels of pain interference, kinesiophobia, anxiety, and depression in patients with PTSS among 463 chronic pain patients referred for treatment. The current sample $(n=682)$ was larger than both studies, thereby emphasizing the findings. Further, the findings are in alignment with existing studies demonstrating that PTSS in chronic pain patients is associated with a more severe symptomatology compared with patients without PTSS. ${ }^{1,7-10}$ However, a few studies only found this group difference for psychosocial and not physical disability, ${ }^{1,10}$ with the current study finding it for both. Taken together, the present results endorse the many studies in the area which found that chronic pain patients with comorbid PTSS posit a more severe pain-related symptomatology, but in addition to this, they also clarify and elaborate these results as it seems that it is the trauma reaction that is related to a worsened symptomatology and not the mere presence of a trauma, as also underlined by Ruiz-Párraga and López-Martínez ${ }^{11}$ and Åkerblom et al. ${ }^{12}$ This is similar to the perspective on development and maintenance of pain presented by the fearavoidance model, ${ }^{30}$ where it is also the negative interpretation of pain rather than the mere presence of pain itself that causes a vicious, negative course, and further in line with the clinical knowledge that it is not the mere existence of a trauma or pain, but rather their functional impact that is of importance.

\section{The moderating effects of PTSS}

PTSS significantly moderated the effects of pain intensity on pain-related psychosocial disability, depression, and anxiety. These interrelations of PTSS and pain are in line with multiple studies in the area using different designs. ${ }^{31-35}$ On the contrary, we did not find evidence of moderation of PTSS on the association between pain and physical disability. This was unexpected as it is not in agreement with the theoretical perspective of the mutual maintenance model ${ }^{4}$ where it would be expected that PTSS, when present, would have a negative impact on pain's associations with both distress and disability. However, it is in agreement with findings by Palyo and Beck, ${ }^{36}$ who found that PTSS and pain intensity were 
both associated with psychosocial impairment, while only pain intensity was associated with physical impairment. This indicates that PTSS moderates the associations between pain and the more psychosocial dimensions of the pain experience (psychosocial disability, depression, and anxiety), while the more rigorous relationship between pain and physical disability is not affected by the presence of PTSS. Hence, the negative effects of PTSS on pain and related outcomes as proposed by the mutual maintenance model ${ }^{4}$ seemingly only apply to certain facets of pain outcomes. This may illustrate that pain prominently features a physical component involving the sensation in the body, which is very much related to physical abilities, while PTSS may be more entangled in the more psychological elements. Also, it may illustrate that the included psychosocial variables are different manifestations of a broader meta-construct such as general psychosocial distress, with PTSS playing a more significant role in the relationships between pain and these manifestations, but it may at the same time also be indicative of a large conceptual overlap between these more psychosocial factors as also indicated by moderate correlations between all psychosocial variables. This is important to take critical notice of in the interpretation of the findings, as a moderating effect of PTSS between pain and pain-related psychosocial outcomes becomes potentially redundant, if all variables represent the same construct. Importantly, however, the inter-correlations are not high enough to speak of multicollinearity per se $(>0.80-90) .{ }^{28}$

In further interpretation, it is important to note the lack of information about the nature of the traumatic experience and potential associations with the onset of pain. Considering PTSS as a moderator in the relationships between pain-related variables using the mutual maintenance perspective may rely on the assumption that the onset of PTSS and pain cooccurred and that PTSS thereby feeds into the pain condition and its associations with disability and distress. However, as information on the causes for pain and PTSS was not collected in the current study, the lack of such co-development could influence interpretation of the results. However, PTSS are likely to have a negative impact on the pain condition and related disability and distress, also when not caused by the same trauma, for example, by affecting the degree of cognitive resources to develop adaptive coping strategies for the pain condition as also suggested by Sharp and Harvey. ${ }^{4} \mathrm{~A}$ study testing the mutual maintenance model would need to ensure that the conditions co-arose after the same trauma for some of the suggested mechanisms to be relevant, eg, pain serving as a persistent reminder of a trauma and thereby feeding into the intrusion-cluster causing hyperarousal.

\section{Clinical implications}

The present findings may be of relevance to pain rehabilitation programs in several ways. First of all, the current results indicate that PTSS may be a relevant marker of specific patient profiles needing specialized treatment as one fifth is reporting clinical levels of PTSS, also presenting a more severe profile with higher levels of pain, pain-related disability, depression, and anxiety. Likewise, the current study shares some important insights into how PTSS may influence the associations between pain and psychosocial disability and distress. Therefore, screening for PTSS in these settings is recommended in order to be able to directly address PTSS when relevant. Indeed, our results point to a similar conclusion as the one outlined by Roth et al. ${ }^{13}$ that there is a profound need to address unresolved PTSS in chronic pain rehabilitation, maybe also in order to gain improvement in other pain-related outcomes.

\section{Limitations}

This study has several limitations. Firstly, the cross-sectional design is a limitation as conclusions on causality cannot be made. Secondly, it is a limitation that PTSS was assessed by the PTSD-8 questionnaire, as there overlaps exist in symptoms of pain and PTSS that may cause pain symptoms to inflate PTSS-ratings and create false positives in relation to PTSS. A clinician-administered diagnostic interview would have minimalized this problem and also allowed for the use of the term PTSD rather than PTSS. However, PTSD-8 has been validated for use among chronic pain patients and designed to focus on the core items of PTSD, which are less prone to this inflation of pain and other disorders, ${ }^{23,25}$ and the PTSD-8 was also recently validated against a diagnostic interview and showed good overall accuracy. ${ }^{25}$ Similarly, there could be a substantial overlap of symptoms tested using the questionnaires used in this study. Individuals with pain are likely to qualify as having relevant symptoms on eg, a depression scale without that being the case (false-positive cases) as the items are not adapted to pain patients, which is important to take critical note of in interpretation of the results.

Thirdly, more in-depth operationalization and details of the traumatic event would have strengthened the study as this would have helped ensure that the event reported was indeed a traumatic event as defined by the criteria. Additionally, it would have strengthened the findings greatly if it was known whether the same trauma caused both the pain condition and the PTSS, as the interaction patterns are likely to be different. ${ }^{5}$ One of the very similar studies in the field selected participants based on this criterion, ${ }^{11}$ which will be good to replicate in future studies. 
Fourthly, as the current study did not seek to test the mutual maintenance model per se, only the influences of PTSS on pain variables were investigated and not vice versa. Future studies should test the full model empirically and the reciprocality of the conditions in studies designed for this purpose. In line with this, the current study did not enable further investigation of the mechanisms of importance in explaining the role of PTSS in the tested pain-related variables. Using the theoretical perspectives of the fear-avoidance model ${ }^{30}$ and the mutual maintenance perspective, ${ }^{4}$ it could be due to a range of cognitive-behavioral factors that amplify the reaction causing an individual with chronic pain and comorbid PTSS to react more severely, such as fear-avoidance variables. ${ }^{37-40}$ Similarly, specific clusters of PTSS may play a specific role in affecting pain's associations with disability and distress, and indeed, a number of studies have pointed to hyperarousal as an important variable in relation to pain and related disability. ${ }^{38,41-45}$ Analysis of some of these factors could have strengthened the findings and should be considered in future studies.

Lastly, the lack of information on the pain diagnosis, medications, and other specific treatments received such as psychotherapy is a limitation as these may serve as relevant covariates. However, Andersen et al. ${ }^{1}$ found that pain diagnosis was not associated with the PTSS group in a study on the same population. Specific interventions targeting the outcomes of interest, on the other hand, may have important impact on the relationships under investigation in the current paper. For example, an earlier study found that approximately half the patients entering this specific pain center used opioids at the time of entry. ${ }^{46}$ Future studies ought to include such information in similar studies.

\section{Conclusion}

The current study adds insights into the associations between PTSS and pain-related outcomes among chronic pain patients referred to pain rehabilitation in a large, mixed chronic pain sample. Because chronic pain patients with comorbid PTSS present a more severe and complex symptom profile and because PTSS changes the associations between pain and several outcomes, it is important to recognize the importance of PTSS in these settings. Therefore, screening and treating PTSS directly is of great importance. However, whether specific targeting of PTSS changes pain-related outcomes needs to be tested in the context of multidisciplinary pain rehabilitation.

\section{Disclosure}

The authors report no conflicts of interest in this work.

\section{References}

1. Andersen TE, Andersen LC, Andersen PG. Chronic pain patients with possible co-morbid post-traumatic stress disorder admitted to multidisciplinary pain rehabilitation - a 1-year cohort study. Eur J Psychotraumatol. 2014;5(1):1-9.

2. Andersen TE, Andersen PG, Vakkala MA, Elklit A. The traumatised chronic pain patient - prevalence of posttraumatic stress disorder PTSD and pain sensitisation in two Scandinavian samples referred for pain rehabilitation. Scand J Pain. 2012;3(1):39-43.

3. American Psychiatric Association. Diagnostic and Statistical manual of mental disorder: DSM-IV-TR. 4th ed. Washington, DC: American Psychiatric Association; 2000.

4. Sharp TJ, Harvey AG. Chronic pain and posttraumatic stress disorder: mutual maintenance? Clin Psychol Rev. 2001;21(6):857-877.

5. Beck JG, Clapp JD. A different kind of co-morbidity: understanding posttraumatic stress disorder and chronic pain. Psychol Trauma. 2011;3(2):101-108.

6. Brennstuhl M, Tarquinio C, Montel S. Chronic pain and PTSD: evolving views on their comorbidity. Perspect Psychiatr Care. 2015;51(4):295-304.

7. Geisser ME, Roth RS, Bachman, JE, Eckert TA. The relationship between symptoms of post-traumatic stress disorder and pain, affective disturbance and disability among patients with accident and nonaccident related pain. Pain. 1996;66(2):207-214.

8. Häuser W, Galek A, Erbslöh-Möller B, et al. Posttraumatic stress disorder in fibromyalgia syndrome: Prevalence, temporal relationship between posttraumatic stress and fibromyalgia symptoms, and impact on clinical outcome. Pain. 2013;154(8):1216-1223.

9. Pedler A, Sterling M. Patients with chronic whiplash can be subgrouped on the basis of symptoms of sensory hypersensitivity and posttraumatic stress. Pain. 2013;154(9):1640-1648.

10. Clapp JD, Masci J, Bennett SA, Beck JG. Physical and psychosocial functioning following motor vehicle trauma: relationships with chronic pain, posttraumatic stress, and medication use. Eur J Pain. 2010;14(4):418-425.

11. Ruiz-Párraga GT, López-Martínez AE. The contribution of posttraumatic stress symptoms to chronic pain adjustment. Health Psychol. 2014;33(9):958-967.

12. Åkerblom S, Perrin S, Rivano Fischer M, McCracken LM. The impact of PTSD on functioning in patients seeking treatment for chronic pain and validation of the posttraumatic diagnostic scale. Int J Behav Med. 2017;24(2):249-259.

13. Roth RS, Geisser ME, Bates R. The relation of post-traumatic stress symptoms to depression and pain in patients with accident-related chronic pain. J Pain. 2008;9(7):588-596.

14. Burris JL, Cyders MA, de Leeuw R, Smith GT, Carlson CR. Posttraumatic stress disorder symptoms and chronic orofacial pain: an empirical examination of the mutual maintenance model. J Orofac Pain. 2009;23(3):243-252.

15. Irwin KC, Konnert C, Wong M, O'Neill TA. PTSD symptoms and pain in Canadian military veterans: the mediating roles of anxiety, depression, and alcohol use. J Trauma Stress. 2014;27(2):175-181.

16. Outcalt SD, Kroenke K, Krebs EE, Chumbler NR, Wu J, Yu Z, Bair MJ. Chronic pain and comorbid mental health conditions: Independent associations of posttraumatic stress disorder and depression with pain, disability, and quality of life. J Behav Med. 2015;38(3): 535-543.

17. Jensen MP, Karoly P, Braver S. The measurement of clinical pain intensity: a comparison of six methods. Pain. 1986;27(1):117-126.

18. McCaffery M, Beebe A. Pain: Clinical Manual for Nursing Practice. Baltimore: Mosby Company; 1993.

19. Cleeland CS, Ryan KM. Pain assessment: global use of the Brief Pain Inventory. Ann Acad Med Singapore. 1994;23(2):129-138.

20. Anagostic C, Gatchel RJ, Mayer TG. The pain disability questionnaire: a new psychometrically sound measure for chronic musculoskeletal disorders. Spine (Phila Pa 1976). 2004;29(20):2290-2302. 
21. Zigmond AS, Snaith RP. The hospital anxiety and depression scale. Acta Psychiatr Scand. 1983;67(6):361-370.

22. Crawford JR, Henry JD, Crombie C, Taylor EP. Normative data for the HADS from a large non-clinical sample. Br J Clin Psychol. 2001;40(Pt 4): 429-434.

23. Hansen M, Andersen TE, Armour C, Elklit A, Palic S, Mackrill T. PTSD-8: a short PTSD inventory. Clin Pract Epidemiol Ment Health. 2010;6:101-108.

24. Maercker A, Brewin CR, Bryant RA, et al. Proposals for mental disorders specifically associated with stress in the International Classification of Diseases-11. Lancet. 2013;381(9878):1683-1685.

25. Andersen TE, Hansen M, Ravn SL, Seehus R, Nielsen MR, Vaegter HB. Validation of the PTSD-8 scale in chronic pain patients. Pain Med. Epub 2017 Jul 13.

26. Collins LM, Schafer JL, Kam CM. A comparison of inclusive and restrictive strategies in modern missing data procedures. Psychol Methods. 2001;6(4):330-351.

27. Bunting BP, Adamson G, Mulhall PK. A Monte Carlo Examination of an MTMM Model With Planned Incomplete Data structures. Structural Equation Modeling. 2002;9(3):369-389.

28. Field A. Discovering Statistics using IBM SPSS Statistics. 4th ed. London: Sage Publications Ltd; 2013.

29. Hayes AF. Introduction to Mediation, Moderation, and Conditational Process Analysis: A Regression-Based Approach. NY: The Gilford Press; 2013.

30. Vlaeyen JW, Linton SJ. Fear-avoidance and its consequences in chronic musculoskeletal pain: a state of art. Pain. 2000;85(3):317-332.

31. Jenewein J, Wirmann L, Moergeli M, Creutzig J, Schnyder U. Mutual influence of posttraumatic stress disorder symptoms and chronic pain among injured accident survivors: a longitudinal study. JTrauma Stress. 2009;22(6):540-548.

32. Kongsted A, Bendix T, Querama E, Kasch H, Bach FW, Korsholm L, Jensen TS. Acute stress response and recovery after whiplash injuries. A one-year prospective study. Eur J Pain. 2008;12(4):455-463.

33. Phifer J, Skelton K, Weiss T, et al. Pain symptomatology and pain medication use in civilian PTSD. Pain. 2011;152(10):2233-2240.

34. Sterling M, Jull G, Viccnzino B, Kenardy J, Darrnell R. Physical and psychological factors predict outcome following whiplash injury. Pain. 2005;114(1-2):141-148.

35. Stratton KJ, Clark SL, Hawn SE, Amstadter AB, Cifu DX, Walker WC. Longitudinal interactions of pain and posttraumatic stress disorder symptoms in U.S. Military Service members following blast exposure. J Pain. 2014;15(10):1023-1032.
36. Palyo SA, Beck JG. Post-traumatic stress disorder symptoms, pain, and perceived life control: associations with psychosocial and physical functioning. Pain. 2005;117(1-2):121-127.

37. Andersen TE, Karstoft KI, Brink O, Elklit A. Pain-catastrophizing and fear-avoidance beliefs as mediators between post-traumatic stress symptoms and pain following whiplash injury - a prospective cohort study. Eur J Pain. 2016;20(8):1241-1252.

38. López-Martínez AE, Ramírez-Maestre C, Esteve R. An examination of the structural link between post-traumatic stress symptoms and chronic pain in the framework of fear-avoidance models. Eur J Pain. 2014;18(8):1129-1138

39. Carty J, O’Donnell M, Evans L, Kazantzis N, Creamer M. Predicting posttraumatic stress disorder symptoms and pain intensity following severe injury: the role of catastrophizing. Eur J Psychotraumatol. 2011;2(1):1-10

40. Martin AL, Halket E, Asmundson GJ, Flora DB, Katz J. Posttraumatic stress symptoms and the diathesis-stress model of chronic pain and disability in patients undergoing major surgery. Clin J Pain. 2010;26(6):518-527.

41. Buitenhuis J, de Jong PJ, Jaspers JP, Groothoff JW. Catastrophizing and causal beliefs in whiplash. Spine (Phila Pa 1976). 2008;33(22): 2427-2433.

42. Cho SK, Heiby EM, McCracken LM, Moon DE, Lee JH. Daily functioning in chronic pain: study of structural relations with posttraumatic stress disorder symptoms, pain intensity, and pain avoidance. Korean J Pain. 2011;24(1):13-21.

43. Cyders MA, Burris JL, Carlson CR. Disaggregating the relationship between posttraumatic stress disorder symptom clusters and chronic orofacial pain: implications for the prediction of health outcomes with PTSD symptom clusters. Ann Behav Med. 2011;41(1):1-12.

44. Liedl A, O'Donnell M, Creamer M, Silove D, McFarlane A, Knaevelsrud C, Bryant RA. Support for the mutual maintenance of pain and post-traumatic stress disorder symptoms. Psychol Med. 2010;40(7):1215-1223.

45. Ritchie C, Hendrikz J, Kenardy J, Sterling M. Derivation of clinical prediction rule to identify both chronic moderate/severe disability and full recovery following whiplash injury. Pain. 2013;154(10):2198-2206.

46. Vaegter HB, Graven-Nielsen T. Pain modulatory phenotypes differentiate subgroups with different clinical and experimental pain sensitivity. Pain. 2016;157(7):1480-1488.
Journal of Pain Research

\section{Publish your work in this journal}

The Journal of Pain Research is an international, peer reviewed, open access, online journal that welcomes laboratory and clinical findings in the fields of pain research and the prevention and management of pain. Original research, reviews, symposium reports, hypothesis formation and commentaries are all considered for publication.

\section{Dovepress}

The manuscript management system is completely online and includes a very quick and fair peer-review system, which is all easy to use. Visit http://www.dovepress.com/testimonials.php to read real quotes from published authors. 